\title{
MYCN downregulates integrin $\alpha 1$ to promote invasion of human neuroblastoma cells
}

\author{
NATSUMI TANAKA and MASAHIRO FUKUZAWA
}

\author{
Department of Pediatric Surgery, Graduate School of Medicine, \\ Osaka University, 2-2, Yamadaoka, Suita, Osaka 565-0871, Japan
}

Received May 28, 2008; Accepted July 21, 2008

DOI: 10.3892/ijo_00000069

\begin{abstract}
Neuroblastoma is a childhood tumor thought to arise through improper differentiation of neural crest cells. MYCN amplification is a prognostic factor that indicates a highly malignant disease and poor patient prognosis. Integrins are important regulators of neuroblastoma attachment and migration and participate in many aspects of metastasis. However, the role of integrins in neuroblastoma metastasis, the leading cause of death from this disease, remains less well understood. Screening of neuroblastoma cell lines for integrin mRNA expression showed that integrin $\alpha 1$ expression was higher in lines such as SK-N-SH and NB69 that do not have MYCN amplification than in cell lines such as IMR32, NB1, NB9 and NB19 that have MYCN amplification. A knockdown of MYCN in NB1 and NB19 cells resulted in increased expression of integrin $\alpha 1$, which correlated with enhanced attachment to the extracellular matrix and reduced migratory activity. In contrast, the overexpression of MYCN in SK-N-SH and NB69 cells resulted in decreased expression of integrin $\alpha 1$, which correlated with reduced attachment to the extracellular matrix and enhanced migratory activity. These results show that MYCN may limit cell adhesion to the extracellular matrix and promote cell migration by downregulating integrin $\alpha 1$.
\end{abstract}

\section{Introduction}

Neuroblastoma is a pediatric tumor of the peripheral nervous system and arises from primordial neural crest cells $(1,2)$. Neuroblastic tumors account for 8-10\% of all pediatric cancers and are second in incidence among solid tumors in children $(2,3)$. Neuroblastoma metastases form through dissemination of transformed cells into the bloodstream and local invasion into bone, leptomeninges and other organs (3-5). Despite a significant improvement in our understanding of the heterogeneous nature of primary neuroblastoma, metastasis,

Correspondence to: Dr Masahiro Fukuzawa, Department of Pediatric Surgery, Graduate School of Medicine, Osaka University, 2-2, Yamadaoka, Suita, Osaka 565-0871, Japan

E-mail: fukuzawa@pedsurg.med.osaka-u.ac.jp

Key words: integrin $\alpha 1$, migration, MYCN, neuroblastoma which is the leading cause of death due to this cancer, remains less well understood (6).

Among the prognostic indicators of neuroblastoma, $M Y C N$ amplification is strongly associated with advanced disease, rapid tumor progression and poor outcome (7). The normal single-copy locus of $M Y C N$ has been mapped to the short arm of chromosome 2p23-24 (8) and encodes a transcription factor that regulates gene expression during cell differentiation and growth $(9,10)$. We recently reported that MYCN regulates cell growth and differentiation in a neuroblastoma cell line with MYCN amplification (11).

Control of cell adhesion is important in a number of biological phenomena, including embryonic development and tumor cell invasion and metastasis (12-14). Integrins are the major metazoan mediators of cell adhesion to extracellular matrix proteins and in vertebrates, play important roles in certain cell-cell adhesions. In addition, integrins make transmembrane connections to the cytoskeleton and activate many intracellular signaling pathways. The mammalian integrin family comprises of $18 \alpha$ and $8 \beta$ subunits, known to assemble into 24 distinct integrins (12). Previous data suggest that integrins are important regulators of neuroblastoma progression (15-18). Nevertheless, the precise effects of MYCN and integrins on neuroblastoma metastasis remain unclear.

The aim of this study was to elucidate the mechanism of MYCN and integrins in tumor metastasis by investigating whether a reduction of MYCN expression could control tumor attachment and migration in neuroblastoma by regulating integrins. Here, we demonstrate that a knockdown of MYCN in neuroblastoma cells harboring $M Y C N$ amplifications increases integrin $\alpha 1$ expression, enhances cell attachment and inhibits migration.

\section{Materials and methods}

Cell culture. Human neuroblastoma cell lines were obtained from the Health Science Research Resources Bank and the RIKEN Cell Bank. NB1, NB9, NB19 and NB69 cells were maintained in RPMI-1640 and IMR-32 and SK-N-SH cells were maintained in MEM Alpha supplemented with $10 \%$ fetal bovine serum (FBS), $100 \mathrm{IU} / \mathrm{ml}$ penicillin and $100 \mu \mathrm{g} / \mathrm{ml}$ streptomycin.

RNA interference. The siRNAs for human MYCN and the control (siTrio-negative control siRNA) were obtained from 
B-Bridge International Inc., Sunnyvale, CA, USA. The sequences of the siRNAs targeting human $M Y C N$ were 5'-CGG AGAUGCUGCUUGAGAA-3', 5'-CGGAGUUGGUAAAG AAUGA-3' and 5'-CAGCAGUUGCUAAAGAAAA-3'. The siTrio-negative control siRNAs were 5'-ATCCGCGCGATA GTACGTA-3', 5'-TTACGCGTAGCGTAATACG-3' and 5'-TATTCGCGCGTATAGCGGT-3'. Transfections were performed with a mixture of all three oligonucleotides, using HiperFect transfection reagent following the manufacturer's instructions (Qiagen Inc., Valencia, CA, USA), $48 \mathrm{~h}$ before cell attachment assays or migration assays.

DNA constructs. A MYCN expression plasmid was constructed by subcloning a human MYCN cDNA into the pCS2+ vector (DNAFORM, Yokohama, Kanagawa, Japan)

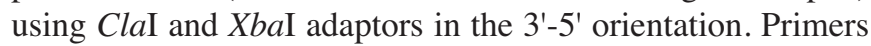
for cloning MYCN were 5'-ATCCCATCGATCACCATGC CGAGCTGCTCCACGTCC-3' and 5'-TGATCTAGACTAG CAAGTCCGAGCGTGTTCAATTTTC-3'. Transfections of plasmids were performed with Lipofectamine 2000 following the manufacturer's instructions (Invitrogen, Carlsbad, CA, USA), $24 \mathrm{~h}$ before cell attachment assays or migration assays. Equal amounts of DNA were used for each transfection.

Conventional PCR. Conventional PCR amplification was carried out at $95^{\circ} \mathrm{C}$ for $1 \mathrm{~min}$, followed by 25 cycles of $95^{\circ} \mathrm{C}$ for $30 \mathrm{sec}, 55^{\circ} \mathrm{C}$ for $30 \mathrm{sec}$ and $72^{\circ} \mathrm{C}$ for $1 \mathrm{~min}$. The primers used for PCR were: $M Y C N, 5^{\prime}$-CCACCAGCAGCACAACT ATG-3' (sense), 5'-GTCCGAGCGTGTTCAATTTT-3' (antisense); integrin $\alpha 1,5^{\prime}$-GCATGACAGTCAGCACT CGT-3' (sense), 5'-TTCAAAAGCTGCTCAGCAAA-3' (antisense); GAPDH, 5'-TGAAGGTCGGAGTCAACGGAT-3' (sense), 5'-CATGTGGGCCATGAGGTCCAC-3' (antisense).

Real-time PCR. Real-time PCR was carried out using the ABI Prism 9700 Sequence Detector System (Perkin-Elmer Applied Biosystems, Foster City, CA, USA). For quantification, $G A P D H$ transcript served as the control and each sample was normalized to its $G A P D H$ transcript level. The primer and probe mixtures for $M Y C N$, integrin $\alpha 1$ and $G A P D H$ were purchased from Perkin-Elmer Applied Biosystems and the PCR method was as recommended in the manufacturer's protocol.

Western blotting. Cells were homogenized in RIPA lysis buffer (Upstate, Lake Placid, NY, USA) and incubated for $10 \mathrm{~min}$ on ice. After centrifugation at $13,000 \mathrm{x} \mathrm{g}$ for $10 \mathrm{~min}$ at $4^{\circ} \mathrm{C}$, supernatants were collected as protein samples. For Western blotting of MYCN and Actin, protein was separated by SDS-PAGE using $10 \%$ polyacrylamide gels and electroblotted onto PVDF membranes (Bio-Rad, Hercules, CA, USA). After blocking of non-specific binding for $1 \mathrm{~h}$ with $5 \%$ non-fat milk in PBS containing $0.1 \%$ Tween-20, membranes were incubated for $1 \mathrm{~h}$ at room temperature with antihuman MYCN antibody (Santa Cruz, Santa Cruz, CA, USA) or antihuman actin antibody (BD Biosciences, San Jose, CA, USA). Membranes were then washed three times with PBS containing $0.1 \%$ Tween-20, incubated with horseradish peroxidase conjugated anti-mouse antibody (Promega
Corporation, Madison, WI, USA) at room temperature and washed three times with PBS containing 0.1\% Tween-20. The immunoblots were developed with an enhanced chemiluminescence system (Amersham Biosciences Inc., Piscataway, NJ, USA). Immunoblotting with antibody to actin provided an internal control for equal protein loading. Densitometry was performed by measuring pixel density in a defined region of each band using Imaging System for Chemiluminescence (AlphaInotech, San Francisco, CA, USA). Blots shown are representative of three independent experiments.

Attachment assay. Attachment assays were carried out using 24-well plates coated with laminin, collagen type IV, or fibronectin (BD Biosciences). Cells $\left(1 \times 10^{4}\right)$ were plated on each well, incubated in the presence of $10 \%$ FBS for $20 \mathrm{~min}$ with or without $3 \mu \mathrm{g} / \mathrm{ml}$ integrin $\alpha 1$ antibody (Chemicon International, Temecula, CA, USA) and rinsed three times with phosphate-buffered saline. Cells that attached to the bottom of the well were fixed with methanol, stained with hematoxylin and eosin and counted under a microscope.

In vitro transwell migration assay. In vitro transwell migration assays were performed using $8.0-\mu \mathrm{m}$ pore size Costar Transwell inserts (Corning Inc., Lowell, MA, USA), according to the manufacturer's protocol. Cells $\left(5 \times 10^{4}\right)$ were added to each well and incubated in the presence of $10 \%$ FBS for $24 \mathrm{~h}$ with or without $3 \mu \mathrm{g} / \mathrm{ml}$ integrin $\alpha 1$ antibody (Chemicon International). Cells that migrated to the bottom of the transwell membrane were fixed with methanol, stained with hematoxylin and eosin and counted under a microscope.

\section{Results}

High expression of integrin al mRNA in neuroblastoma cell lines without MYCN amplifications. To test whether integrins are involved in the metastatic phenotype of neuroblastoma cells, we analyzed integrin (integrin $\alpha 1,2,3,4,5, v$, integrin $\beta 1,3$ and 5) mRNA levels in neuroblastoma cell lines with MYCN amplifications, which express high levels of MYCN (IMR32, NB1, NB9 and NB19) or without $M Y C N$ amplifications (SK-N-SH and NB69). Among the integrins we screened, the expression of integrin $\alpha 1$ was relatively high in cell lines lacking MYCN amplification (Fig. 1a), as confirmed by real-time PCR (Fig. 1b).

MYCN-siRNA upregulates integrin al expression in NB1 and NB19 neuroblastoma cells. We then generated MYCNknockdown NB1 or NB19 cells using siRNA targeting $M Y C N$ and analyzed integrin (integrin $\alpha 1,2,3,4,5, v$, integrin $\beta 1,3$ and 5) expression. RT-PCR and real-time PCR analysis of MYCN mRNA in MYCN-siRNA transfected NB1 or NB19 cells showed transcript depletion (Fig. 2a and 2b). RT-PCR and real-time PCR analysis showed that the knockdown of MYCN in neuroblastoma cells harboring MYCN amplifications increased integrin al mRNA expression (Fig. 2a and b). In addition, Western blotting and densitometry analysis showed that MYCN protein was depleted in these cells (Fig. 3a and b). These results are consistent with a previous finding that the 
a
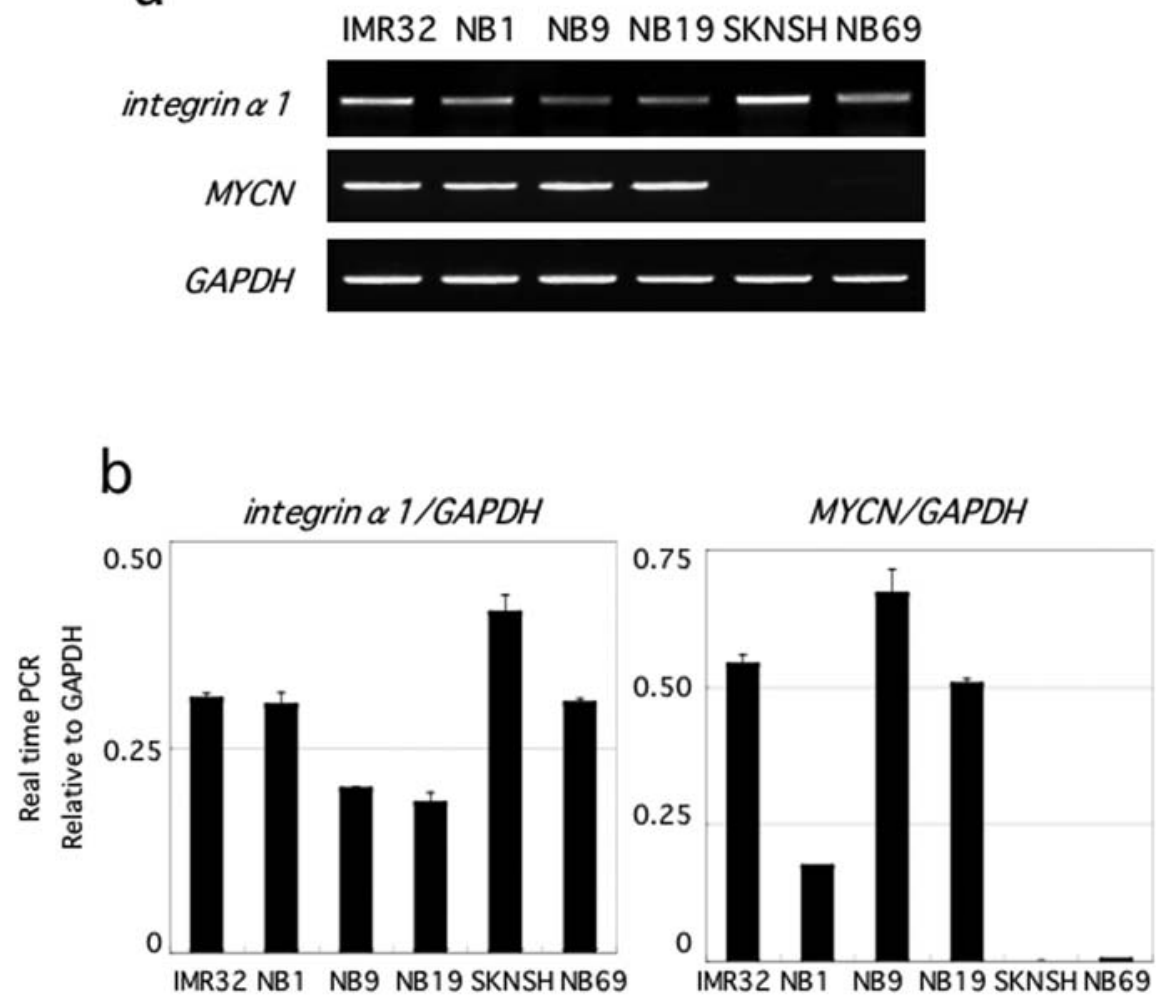

Figure 1. MYCN and integrin $\alpha 1$ mRNA expression in neuroblastoma cell lines. (a) Integrin al and MYCN mRNA expression were evaluated by semiquantitative reverse transcription PCR in neuroblastoma cell lines (IMR32, NB1, NB9 and NB19) harboring MYCN amplifications and in neuroblastoma cell lines without such amplifications (SK-N-SH and NB69). (b) The relative mRNA expression of integrin al and MYCN in these six neuroblastoma cell lines was evaluated by real-time PCR. Error bars represent the standard deviations of three replicates.

a

siRNA $\frac{\text { NB1 }}{\text { Cont.MYCN }} \frac{\text { NB19 }}{\text { Cont.MYCN }}$

integrin $\alpha 1$
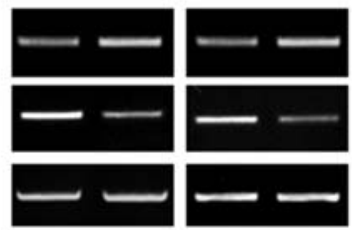

MYCN

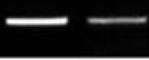

GAPDH

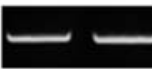

b

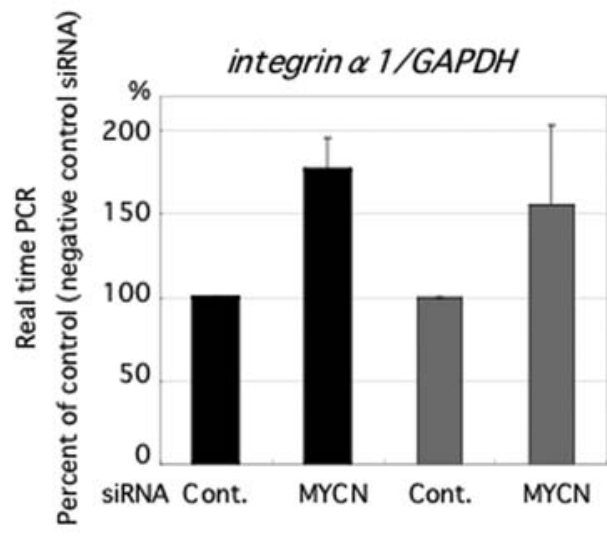

$\%$

$M Y C N / G A P D H$

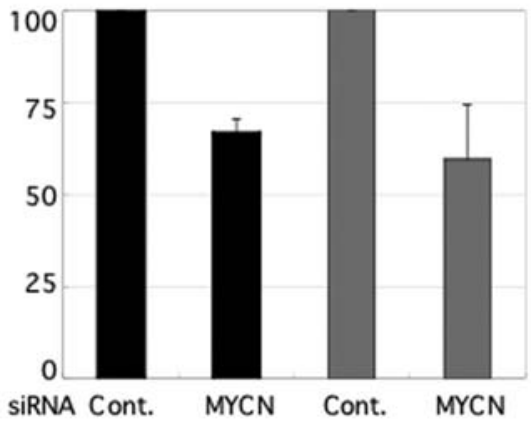

NB1

NB19

Figure 2. MYCN and integrin al mRNA expression in NB1 and NB19 cells expressing control- or MYCN-siRNA. (a) Semiquantitative reverse transcription PCR and (b) real-time PCR show the expression of MYCN and integrin al. Elevation of integrin al and depletion of MYCN expression were observed in neuroblastoma cells transfected with MYCN-siRNA compared with cells transfected with control-siRNA. Error bars represent the standard deviations of five replicates. 

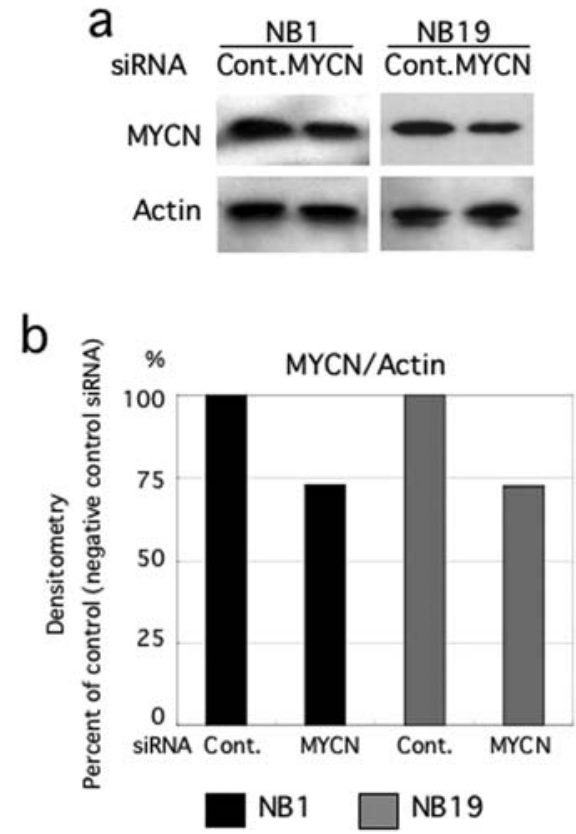

Figure 3. MYCN protein levels in NB1 and NB19 cells expressing control- or MYCN-siRNA. (a) MYCN protein levels were evaluated by Western blot analysis and (b) densitometry. Each figure shows a representative result of at least three experiments.

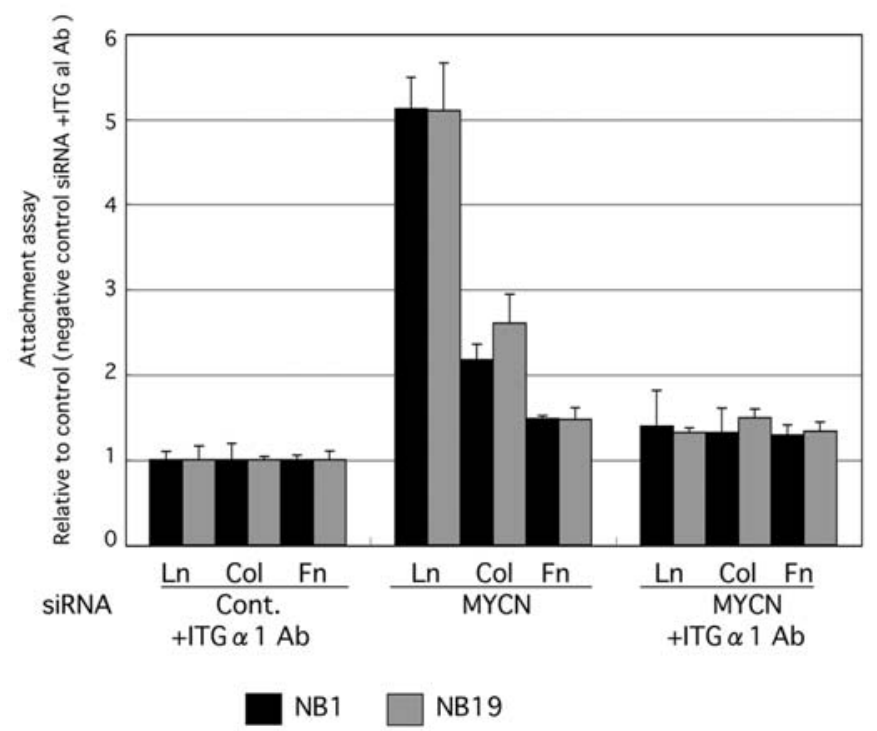

Figure 4. The effects of MYCN knockdown on neuroblastoma cell attachment. NB1 and NB19 cells expressing control- or MYCN-siRNA were incubated on 24-well plates coated with laminin, collagen type IV, or fibronectin with or without $3 \mu \mathrm{g} / \mathrm{ml}$ integrin $\alpha 1$ antibody for $20 \mathrm{~min}$ during attachment assays. Error bars represent the standard deviations of three replicates (Ln, laminin; Col, collagen type IV and Fn, fibronectin).

expression of integrin $\alpha 1$ is associated with the morphological differentiation of neuroblastoma cell lines $(17,18)$ and suggests that integrin $\alpha 1$ may be an important regulator of human neuroblastoma progression.

MYCN-siRNA enhances adhesion of NB1 and NB19 cells to the extracellular matrix by upregulating integrin $\alpha 1$. We then investigated whether MYCN influences cellular adhesion to

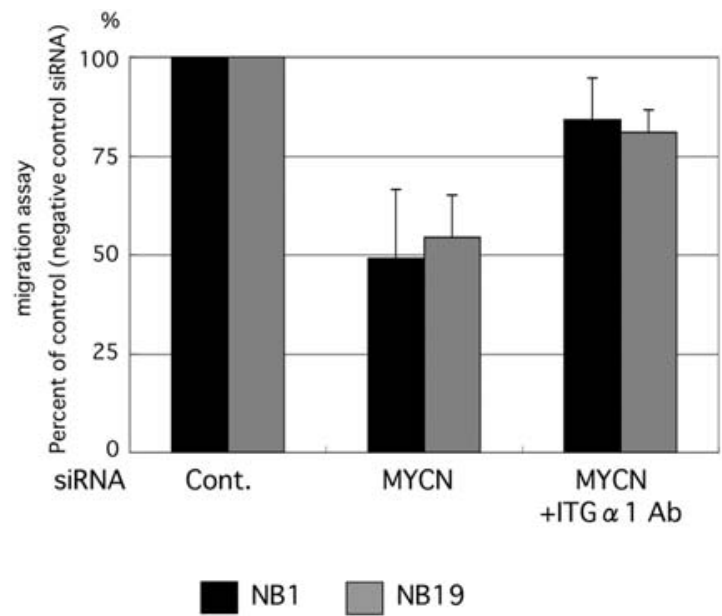

Figure 5. The effects of MYCN knockdown on neuroblastoma cell migration. NB1 and NB19 cells expressing control- or MYCN-siRNA were incubated with or without $3 \mu \mathrm{g} / \mathrm{ml}$ integrin $\alpha 1$ antibody for $24 \mathrm{~h}$ during in vitro transwell migration assays. Error bars represent the standard deviations of three replicates.

the extracellular matrix by regulating integrin $\alpha 1$. We performed attachment assays using NB1 and NB19 cells transfected with control- and MYCN-siRNA and treated with integrin $\alpha 1$ antibody. An increased number of MYCN-siRNAtreated cells adhered to laminin, collagen type IV, or fibronectin compared to cells transfected with control-siRNA and treated with integrin $\alpha 1$ antibody (Fig. 4). This enhancement of adhesion to laminin and collagen type IV could be abrogated by treatment with integrin $\alpha 1$ antibody (Fig. 4). As fibronectin is not a ligand for integrin $\alpha 1$, the enhancement of adhesion to fibronectin occurs through an unknown mechanism. However, our results show that integrin $\alpha 1$ was the main factor of neuroblastoma adhesion. These results suggest that inhibition of MYCN resulted in increased integrin $\alpha 1$ and cellular adhesion to the extracellular matrix.

MYCN-siRNA inhibits the in vitro invasive behavior of NBI and NB19 cells by upregulating integrin al expression. To examine whether MYCN and integrin $\alpha 1$ are involved in neuroblastoma migration, we performed in vitro migration assays using MYCN-knockdown NB1 and NB19 cells. In transwell migration assays, control NB1 and NB19 cells showed active migration, reflecting their invasive properties; in contrast, MYCN-knockdown NB1 and NB19 cells exhibited poor migratory activity (Fig. 5). The defect in migratory activity in MYCN knockdown cells was rescued by integrin $\alpha 1$ antibody (Fig. 5). To exclude the possibility of off-target effects, we used a different siRNA obtained from Qiagen. RT-PCR and real-time PCR analysis of MYCN mRNA in MYCN-siRNA transfected NB1 or NB19 cells showed $30 \%$ transcript depletion (data not shown). This partial suppression was similar in migration assays and attachment assays (data not shown). Taken together, these results indicate that MYCN may limit cell adhesion to the extracellular matrix and may promote cell migration by downregulating integrin $\alpha 1$.

MYCN overexpression downregulates integrin al expression in SK-N-SH and NB69 neuroblastoma cells. To confirm this 
a

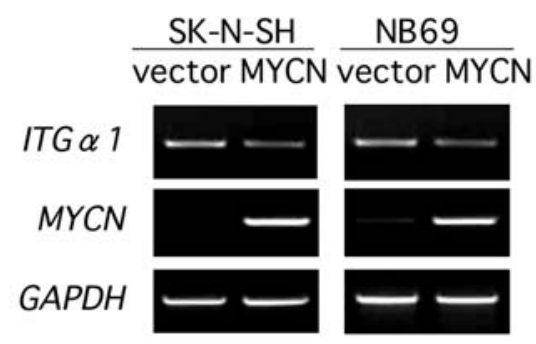

b
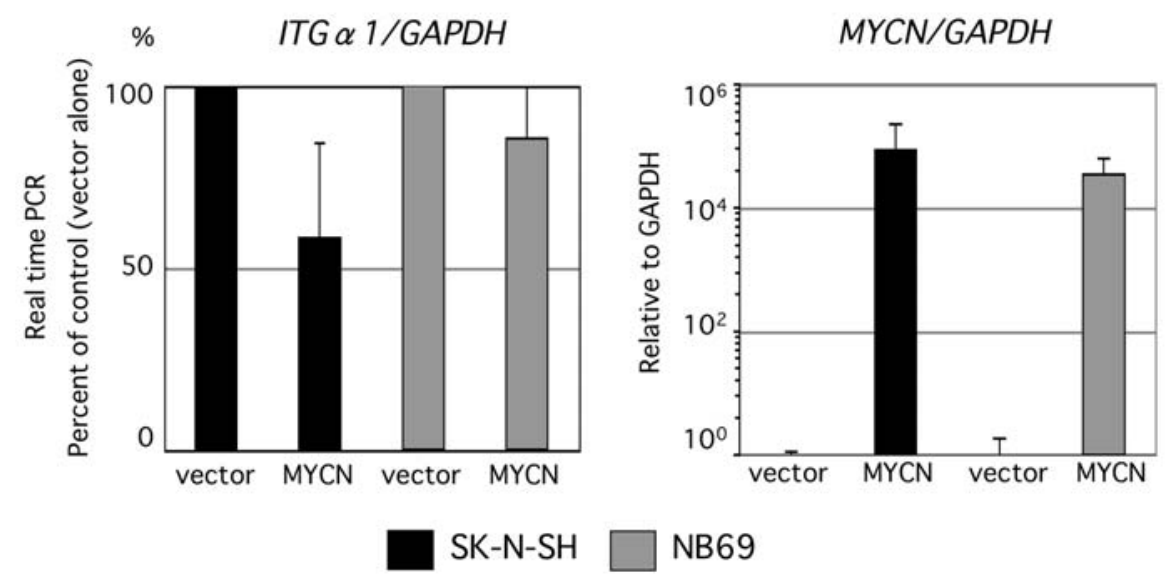

Figure 6. MYCN and integrin al mRNA expression in SK-N-SH and NB69 cells expressing pCS2+ or pCS2+MYCN. (a) Semiquantitative reverse transcription PCR and (b) real-time PCR show the expression of MYCN and integrin al. Depletion of integrin al and elevation of MYCN expression were observed in neuroblastoma cells transfected with pCS2+MYCN compared with cells transfected with vector only. Error bars represent the standard deviations of three replicates.

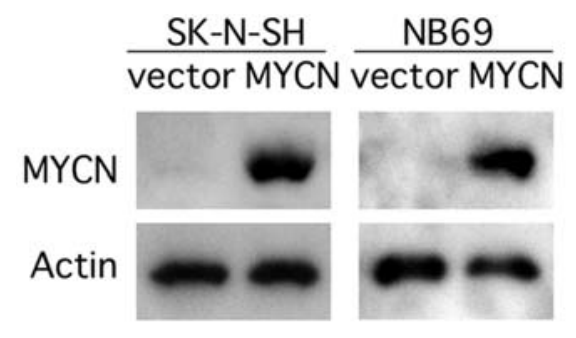

Figure 7. MYCN protein levels in SK-N-SH and NB69 cells expressing pCS2+ or pCS2+MYCN. MYCN protein levels were evaluated by Western blot analysis. Each figure shows a representative result of at least three experiments.

hypothesis, we generated MYCN-overexpressing SK-N-SH and NB69 cells using a MYCN expression vector. RT-PCR and real-time PCR analysis of MYCN mRNA in pCS2+MYCN transfected SK-N-SH and NB69 cells showed transcript enhancement (Fig. 6a and b). RT-PCR and real-time PCR analysis showed that overexpression of MYCN in SK-N-SH and NB69 cells decreased integrin $\alpha 1$ mRNA expression (Fig. 6a and b). In addition, Western blot analysis showed that the MYCN protein was elevated in these cells (Fig. 7).

MYCN overexpression inhibits adhesion and enhances the in vitro invasive behavior of SK-N-SH and NB69 cells by downregulating integrin $\alpha 1$. To determine whether MYCN overexpression can induce opposite effects on cell adhesion and motility, we performed attachment assays and in vitro

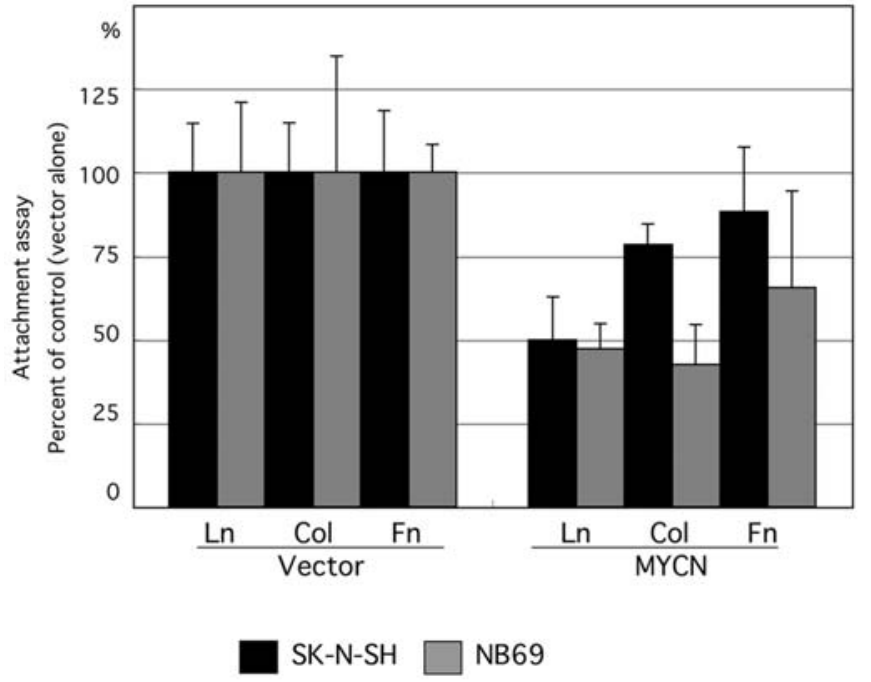

Figure 8. The effects of MYCN overexpression on neuroblastoma cell attachment. SK-N-SH and NB69 cells expressing pCS2+ or pCS2+MYCN were incubated on 24-well plates coated with laminin, collagen type IV, or fibronectin for $20 \mathrm{~min}$ during attachment assays. Error bars represent the standard deviations of three replicates (Ln, laminin; Col, collagen type IV and Fn, fibronectin).

migration assays using SK-N-SH and NB69 cells transfected with $\mathrm{pCS} 2+$ and $\mathrm{pCS} 2+\mathrm{MYCN}$. A decreased number of pCS2+MYCN transfected cells adhered to laminin or collagen type IV compared to cells transfected with pCS2+ (Fig. 8). A decreased number of $\mathrm{pCS} 2+\mathrm{MYCN}$-transfected cells adhered 


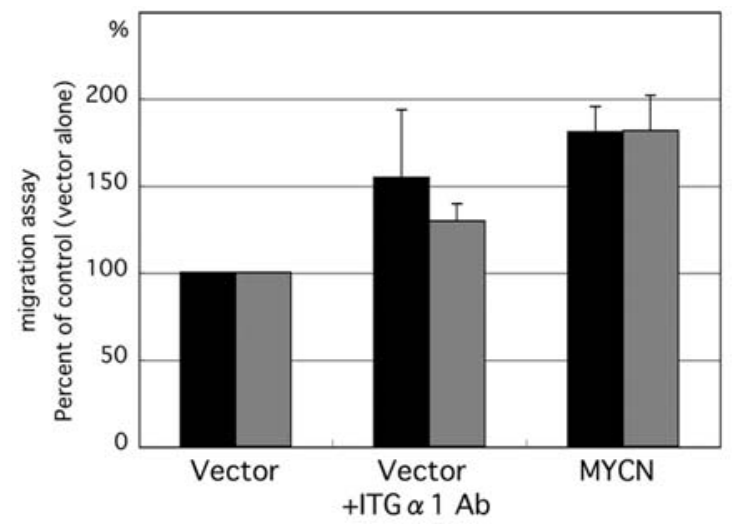

SK-N-SH

NB69

Figure 9. The effects of MYCN overexpression on neuroblastoma cell migration. SK-N-SH and NB69 cells expressing pCS2+ or pCS2+MYCN were incubated with or without $3 \mu \mathrm{g} / \mathrm{ml}$ integrin $\alpha 1$ antibody for $24 \mathrm{~h}$ during in vitro transwell migration assays. Error bars represent the standard deviations of three replicates.

to fibronectin, through an unknown mechanism. In transwell migration assays, increased migration of cells treated with integrin $\alpha 1$ antibody and pCS2+MYCN were observed compared to the control cells (Fig. 9). Thus, downregulation of integrin $\alpha 1$ by MYCN overexpression inhibits adhesion and enhances the in vitro invasive behavior of SK-N-SH and NB69 cells.

Taken together, our results show that MYCN limits cell adhesion to the extracellular matrix and promotes cell migration by downregulating integrin $\alpha 1$.

\section{Discussion}

Neuroblastoma is the most common solid tumor in children (5) and children with metastasis fail to respond to medical intervention $(5,6)$. The only available therapeutic strategies for advanced neuroblastoma include surgical resection, radiation and myeloablative chemotherapy with bone marrow transplantation $(5,19)$. Even with the most intensive treatment, the 2-year relapse-free survival of stage IV neuroblastoma with remote metastasis is only $\sim 40 \%(5,19)$. Neuroblastoma typically spreads to regional lymph nodes, leptomeninges, bone and bone marrow (3-5). Whereas our knowledge of the heterogeneous nature of primary neuroblastoma has significantly improved during recent years, metastasis, the leading cause of death, remains poorly understood (6). Improvements in neuroblastoma patient survival require the identification of molecular targets for treatment based on a thorough understanding of the metastatic process.

Metastasis is a complex and organized process that consists of multiple but interrelated steps, during which tumor cells leave the primary tumor, gain access to the circulatory system, are carried to a distant site, exit the circulation and eventually grow in a different microenvironment (6). Local invasion, the first step of metastasis, requires complex interactions, including recognition and attachment of tumor cells to extracellular matrix binding sites, proteolytic dissolution of the extracellular matrix and tumor cell migration into the surrounding tissue $(6,20)$. Control of cell attachment is therefore important for tumor cell invasion $(12,21,22)$. Integrins are the major metazoan receptors for cell adhesion to extracellular matrix proteins and, in vertebrates, also play important roles in cell-cell adhesion (12). Integrins and their ligands play key roles in development, immune responses, leukocyte traffic, hemostasis and cancer (12). The involvement of some integrins in neuroblastoma progression has also been reported (15-18).

MYCN amplification was identified in neuroblastoma by Schwab et al over 20 years ago (8). Soon after this discovery, $M Y C N$ amplification was confirmed as the most significant prognostic indicator of adverse disease outcome in neuroblastoma (7). However, the association of $M Y C N$ amplification and a more aggressive phenotype is still unclear (5). MYCN is a transcription factor and oncoprotein known to repress several adhesion-related genes, including integrins $(21,22)$. MYCN may modulate neuroblastoma tumorigenicity through interference with integrin-mediated cell adhesion. Our experiments have established that MYCN is essential for the invasive behavior of neuroblastoma cells by downregulating integrin $\alpha 1$. Four lines of evidence support this idea. i) The knockdown of MYCN in neuroblastoma harboring MYCN amplifications resulted in increased integrin $\alpha 1$ expression. ii), High expression of endogenous integrin $\alpha 1$ mRNA was observed in neuroblastoma cell lines that did not harbor MYCN amplifications. iii), MYCN depletion in these cells enhanced attachment and inhibited migratory activity. iv), The enhanced adhesion and defective migration were both abrogated by treatment with integrin $\alpha 1$ antibody. We confirmed these observations by overexpression of MYCN in neuroblastoma cells without MYCN amplifications.

Previous reports have indicated increased tumorigenicity associated with reduced levels of integrin $\alpha 2,3$ and $\beta 1$ caused by MYCN overexpression in neuroblastoma $(17,18,23,24)$. In this study, to elucidate the MYCN-dependent mechanism regulating cell adhesion and motility, we investigated various cell adhesion molecules, including $\mathrm{N}$-cadherin, neural cell adhesion molecule, integrins $\alpha 1-5, \mathrm{~V}$ and $\beta 1,3$ and 5 (data not shown). We found a significant influence of MYCNknockdown on integrin $\alpha 1$; in contrast, integrins $\alpha 2,3$ and $\beta 1$ were unaffected. Although previous reports showed that integrin $\alpha 1$ was associated with neuroblastoma cell differentiation $(17,18)$, this is the first report to demonstrate an invasive role for integrin $\alpha 1$ regulated by $\mathrm{MYCN}$. As fibronectin is not a ligand for integrin $\alpha 1$, the enhancement of adhesion to fibronectin occurred through an unknown mechanism. However, our results suggest that integrin $\alpha 1$ might be key for adhesion and motility of neuroblastoma cells harboring MYCN amplifications.

Our results suggest that MYCN may be exerting a negative regulatory effect on transcription of integrin $\alpha 1$, especially since MYCN is a DNA binding protein. Previous reports have shown that several integrins are influenced by increased levels of MYCN $(23,25)$. However, it is not known whether any of these genes is directly regulated by MYCN binding to the promoter region (25). Thus, more direct transcriptional analyses will be required to test these hypotheses.

Our findings suggest that MYCN promotes metastasis by downregulating integrin $\alpha 1$, representing one mechanism that promotes increased neuroblastoma metastasis. Integrin $\alpha 1$ 
therefore may be a promising molecular target for therapeutic drugs that target neuroblastoma metastasis. Future studies will fully explore the interaction between MYCN and integrins during neuroblastoma cell invasion.

\section{Acknowledgements}

We thank T. Hirano, T. Ueno and T. Oue for their critical comments and helpful suggestions. This study was supported by a Grant-in-Aid (No. 17591861) for scientific research from the Ministry of Education, Culture, Sports, Science and Technology of Japan.

\section{References}

1. Castleberry RP: Biology and treatment of neuroblastoma. Pediatr Clin North Am 44: 919-937, 1997.

2. Cianfarani S and Rossi P: Neuroblastoma and insulin-like growth factor system. New insights and clinical perspectives. Eur J Pediatr 156: 256-261, 1997.

3. Philip T: Overview of current treatment of neuroblastoma. Am J Pediatr Hematol Oncol 14: 97-102, 1992.

4. Tanabe M, Ohnuma N, Iwai J, et al: Bone marrow metastasis of neuroblastoma analyzed by MRI and its influence on prognosis. Med Pediatr Oncol 24: 292-299, 1995.

5. Brodeur GM: Neuroblastoma: biological insights into a clinical enigma. Nat Rev Cancer 3: 203-216, 2003.

6. Tasnim A and Yves AD: Mechanisms of invasion and metastasis in human neuroblastoma. Cancer Metastasis Rev 25: 645-657, 2006.

7. Seeger RC, Brodeur GM, Sather H, et al: Association of multiple copies of the $\mathrm{N}$-myc oncogene with rapid progression of neuroblastomas. N Engl J Med 313: 1111-1116, 1985.

8. Schwab M, Varmus HE, Bishop JM, et al: Chromosome localization in normal human cells and neuroblastomas of a gene related to c-myc. Nature 308: 288-291, 1984.

9. Charron J, Malynn BA, Fisher P, et al: Embryonic lethality in mice homozygous for a targeted disruption of the N-myc gene. Genes Dev 6: 2248-2257, 1992.

10. Stanton BR, Perkins AS, Tessarollo L, Sassoon DA and Parada LF: Loss of N-myc function results in embryonic lethality and failure of epithelial component of the embryo to develop. Genes Dev 6: 2235-2247, 1992.

11. Nara K, Kusafuka T, Yoneda A, Oue T, Sangkhathat S and Fukuzawa M: Silencing $M Y C N$ by RNA interference induces growth inhibition, apoptotic activity and cell differentiation in a neuroblastoma cell line with $M Y C N$ amplification. Int J Oncol 30: 1189-1196, 2007.
12. Richard $\mathrm{OH}$ : Integrins: bidirectional, allosteric signaling machines. Cell 110: 673-687, 2002.

13. Albelda SM: Role of integrins and other cell adhesion molecules in tumor progression and metastasis. Lab Invest 68: 4-17, 1993.

14. Ruoslahti E and Giancotti FG: Integrins and tumor cell dissemination. Cancer Cells 1: 119-126, 1989.

15. Amy M, Cynthia MG, Bhumsoo K, Kenneth LG and Eva LF: Integrin expression regulates neuroblastoma attachment and migration. Neoplasia 6: 332-342, 2004.

16. Judware R and Culp LA: Over-expression of transfected N-myc oncogene in human SKNSH neuroblastoma cells down-regulates expression of beta 1 integrin subunit. Oncogene 11: 2599-2607, 1995.

17. Rossino P, Defilippi P, Silengo L and Tarone G: Up-regulation of the integrin alpha1/beta 1 in human neuroblastoma cells differentiated by retinoic acid: correlation with increased neurite outgrowth response to laminin. Cell Regul 2: 1021-1033, 1991.

18. Rozzo C, Ratti P, Ponzoni M and Cornaglia FP: Modulation of alpha1beta1, alpha2beta1, and alpha3beta1 integrin heterodimers during human neuroblastoma cell differentiation. FEBS Lett 332: 263-267, 1993.

19. Kaneko M, Tsuchida Y, Mugishima $\mathrm{H}$, et al: Intensified chemotherapy increases the survival rates in patients with stage 4 neuroblastoma with $M Y C N$ amplification. J Pediatr Hematol Oncol 24: 613-621, 2002.

20. Liotta LA: Tumor invasion and metastasis-role of the extracellular matrix. Cancer Res 46: 1-7, 1986.

21. Carla G, Shaun MC, Leonard PJ and Robert NE: The Myc/ Max/Mad network and the transcriptional control of cell behavior. Annu Rev Cell Dev Biol 16: 653-699, 2000.

22. Gross N, Balmas BK and Brognara CB: MYCN-related suppression of functional CD44 expression enhances tumorigenic properties of human neuroblastoma cells. Exp Cell Res 260: 396-403, 2000.

23. Judware R and Culp LA: Concominant down-regulation of expression of integrin subunits by $\mathrm{N}$-myc in human neuroblastoma cells: differential regulation of alpha2, alpha3 and beta1. Oncogene 14: 1341-1350, 1997.

24. van Golen CM, Soules ME, Grauman AR and Feldman EL: $\mathrm{N}$-myc overexpression leads to decreased beta 1 integrin expression and increased apoptosis in human neuroblastoma cells. Oncogene 22: 2664-2673, 2003.

25. Thomas WD, Raif A, Hansford L, et al: N-myc transcription molecule and oncoprotein. Int J Biochem Cell Biol 36: 771-775, 2004. 